\title{
Utility of diffusion tensor imaging studies linked to neuronavigation and other modalities in repeat hemispherotomy for intractable epilepsy
}

\author{
Erin N. Kiehna, MD, ${ }^{1}$ Elysa Widjaja, MD, ${ }^{2}$ Stephanie Holowka, MRT(R), ${ }^{2}$ O. Carter Snead III, MD, ${ }^{3}$ \\ James Drake, MBBCh, ${ }^{4}$ Shelly K. Weiss, MD, ${ }^{3}$ Ayako Ochi, MD, ${ }^{3}$ Eric M. Thompson, MD, ${ }^{4}$ \\ Cristina Go, MD, ${ }^{3}$ Hiroshi Otsubo, MD, ${ }^{3}$ Elizabeth J. Donner, MD, ${ }^{3}$ and James T. Rutka, MD, PhD ${ }^{4}$ \\ 'Division of Neurosurgery, Children's Hospital Los Angeles, University of Southern California, Los Angeles, California; and \\ Divisions of ${ }^{2}$ Diagnostic Imaging, ${ }^{3}$ Neurology, and ${ }^{4}$ Pediatric Neurosurgery, Hospital for Sick Children, University of Toronto, \\ Ontario, Canada
}

OBJECTIVE Hemispherectomy for unilateral, medically refractory epilepsy is associated with excellent long-term seizure control. However, for patients with recurrent seizures following disconnection, workup and investigation can be challenging, and surgical options may be limited. Few studies have examined the role of repeat hemispherotomy in these patients. The authors hypothesized that residual fiber connections between the hemispheres could be the underlying cause of recurrent epilepsy in these patients. Diffusion tensor imaging (DTI) was used to test this hypothesis, and to target residual connections at reoperation using neuronavigation.

METHODS The authors identified 8 patients with recurrent seizures following hemispherectomy who underwent surgery between 1995 and 2012. Prolonged video electroencephalography recordings documented persistent seizures arising from the affected hemisphere. In all patients, DTI demonstrated residual white matter association fibers connecting the hemispheres. A repeat craniotomy and neuronavigation-guided targeted disconnection of these residual fibers was performed. Engel class was used to determine outcome after surgery at a minimum of 2 years of follow-up.

RESULTS Two patients underwent initial hemidecortication and 6 had periinsular hemispherotomy as their first procedures at a median age of 9.7 months. Initial pathologies included hemimegalencephaly $(n=4)$, multilobar cortical dysplasia $(n=3)$, and Rasmussen's encephalitis $(n=1)$. The mean duration of seizure freedom for the group after the initial procedure was 32.5 months (range 6-77 months). In all patients, DTI showed limited but definite residual connections between the 2 hemispheres, primarily across the rostrum/genu of the corpus callosum. The median age at reoperation was 6.8 years (range 1.3-14 years). The average time taken for reoperation was 3 hours (range 1.8-4.3 hours), with a mean blood loss of $150 \mathrm{ml}$ (range 50-250 ml). One patient required a blood transfusion. Five patients are seizure free, and the remaining 3 patients are Engel Class II, with a minimum follow-up of 24 months for the group.

CONCLUSIONS Repeat hemispherotomy is an option for consideration in patients with recurrent intractable epilepsy following failed surgery for catastrophic epilepsy. In conjunction with other modalities to establish seizure onset zones, advanced MRI and DTI sequences may be of value in identifying patients with residual connectivity between the affected and unaffected hemispheres. Targeted disconnection of these residual areas of connectivity using neuronavigation may result in improved seizure outcomes, with minimal and acceptable morbidity.

http://thejns.org/doi/abs/10.3171/2015.7.PEDS15101

KEY WORDS epilepsy surgery; hemispherectomy; periinsular hemispherotomy; diffusion tensor imaging; neuronavigation

$\mathrm{H}$ EMISPHERECTOMY for children with unilateral, medically refractory epilepsy is associated with excellent long-term seizure control. Outcomes are dependent on achieving a complete disconnection between the affected, diffusely abnormal hemisphere and the nor- mal hemisphere. For the $15 \%-30 \%$ of children with recurrent seizures following procedures such as periinsular hemispherotomy $(\mathrm{PIH}),{ }^{12}$ regaining seizure control can be problematic-requiring the addition of new anticonvulsant medications, a trial with vagal nerve stimulation, or

ABBREVIATIONS DTI = diffusion tensor imaging; EEG = electroencephalography; HD = hemidecortication; MEG = magnetoencephalography; $\mathrm{PIH}=$ periinsular hemispherotomy; VP = ventriculoperitoneal.

SUBMITTED February 16, 2015. ACCEPTED July 16, 2015

INCLUDE WHEN CITING Published online December 11, 2015; DOI: 10.3171/2015.7.PEDS15101. 
conversion to anatomical hemispherectomy. ${ }^{15,30}$ Whereas the complications of hemispherectomy at the time of initial surgery, whether anatomical or functional, are well described, $, 214,15,20$ issues relating to repeat hemispherotomy are not as well described in the literature.

For children with persistent or recurrent seizures following hemispherectomy, it is imperative that the epilepsy surgery team determines if disconnection of the affected hemisphere has been successfully achieved. In addition to prolonged video electroencephalography (EEG) recordings to demonstrate the semiology of the recurrent/residual seizure pattern, MRI studies should be obtained to carefully demonstrate the completeness of the disconnection. Although this can sometimes be difficult given the complexities of the relevant association, commissural, and projection fiber systems-complexities that are caused by congenital or acquired anatomical deformity in the affected hemisphere-advances in MRI technology have made the determination of extent of disconnection easier to visualize, document, and correlate with the seizure semiology. In this regard, the development of MR fiber tractography in conjunction with diffusion tensor imaging (DTI) has the potential in theory to be used to study persistent white matter fiber tracts connecting the 2 hemispheres in postoperative cases of hemispherectomy for which seizure persistence or recurrence remains problematic.

A DTI investigation is based on the principle that the diffusivity of water molecules is restricted in highly organized tissues, such as white matter tracts, a process known as diffusion anisotropy. ${ }^{16}$ Diffusion anisotropy is influenced by intraaxonal organization, degree of myelination, density of fiber and neuroglial cell packing, and fiber diameter. ${ }^{21}$ Fractional anisotropy is a measure of anisotropy, and quantitative maps of fractional anisotropy can be generated, with higher signal representing greater degree of anisotropy. ${ }^{24}$ Color vector maps reflect both anisotropy and direction of the white matter, with intensity representing the anisotropy and color representing the direction of the white matter. A tensor can be modeled whereby the restricted diffusion is parallel to the direction of the fiber bundles. ${ }^{10}$ Fractional anisotropy and mean diffusivity are measures that are used to quantify tensor-like phenomena so that tractography and DTI maps can be created to estimate the position of relevant white matter fibers.

To date, there have been few studies that have examined the benefit of a comprehensive workup of the pediatric patient with failed hemispherectomy, including the use of DTI, to guide treatment aimed at disconnecting persistent connectivity fibers. ${ }^{3,30}$ Here we present our experience with a subset of patients who were identified as having persistent bands of white matter connectivity between the 2 hemispheres following hemispherectomy and who underwent targeted resection of these bands in attempts to improve their underlying intractable epilepsy. Our neurosurgical technique in these cases is described.

\section{Methods}

\section{Index Hemispheric Procedures Performed for Intractable Epilepsy}

Prior to 1997, the surgical approach to hemispherecto- my at the Hospital for Sick Children, Toronto, was hemidecortication (HD). In 1997, a change in technique was adopted in favor of PIH, which has been used since that time for children with catastrophic, hemispheric epilepsy. Both techniques were described in detail recently., ${ }^{3,12}$ Briefly, HD is performed after a large unilateral craniotomy. The dura mater is typically opened widely, and the affected brain tissue is resected lobe by lobe from the cortex to the white matter, in stages. The Cavitron is used to resect the cortical tissue by aspirating within the white matter planes. In the end, only a small amount of cerebral tissue is left overlying the basal ganglia and deep gray matter structures such as the thalamus. The ventricle is not routinely exposed, and mesiotemporal structures are not resected in cases in which HD is used. $9,17,26$

For PIH, the frontoparietal opercular cortex is resected down to the insular pial bank, creating the suprainsular window; the infrainsular window is created by resecting the temporal opercular cortex down to the pia overlying the insula. ${ }^{31,32}$ Typically, an anterolateral temporal lobectomy including mesial structures is performed in most cases. The corona radiata is then transected following the opening of the lateral ventricular system. A complete callosotomy is performed from within the lateral ventricle, using the pericallosal vessels anteriorly and the free edge of the falx and tentorium posteriorly as delimiting structures. A frontal disconnection is then performed by following the pia along the medial sphenoid wing of the anterior cranial fossa floor to the interhemispheric fissure, and by joining it to the frontal ventricular horn incision. Finally, the insular cortex is resected either en bloc or piecemeal, depending on the position of the penetrating insular cortical arterial candelabra.

\section{Patient Workup and Investigation Following Failed HD or PIH}

This series includes pediatric patients with recurrent seizures following an initial hemispherectomy that was performed between 1995 and 2012. With the recurrence of seizures, all patients were extensively reinvestigated with routine EEG, prolonged video EEG recordings in the epilepsy monitoring unit, and neuroimaging (MRI scaneither 1.5 or $3 \mathrm{~T}$ ). MRI studies included high-resolution volumetric T1-weighted imaging as well as axial and coronal T2-weighted imaging and proton density, FLAIR, and DTI sequences. Color vector maps and fractional anisotropy maps were generated from the DTI.

Magnetoencephalography (MEG) was performed in select cases as previously described. ${ }^{29}$ The results of all of these investigations were discussed at a multidisciplinary pediatric epilepsy surgery conference.

\section{Repeat Neurosurgical Procedure for Recurrent Epilepsy}

In preparation for repeat craniotomy, the volumetric T1-weighted and DTI sequences of all patients were imported into the BrainLab workstation for intraoperative navigation. Identification of the areas of suspected persistent connectivity were outlined and color coded by the neuroradiologist (E.W.). The patient's head was rigidly fixed with a Sugita headrest for frameless stereotactic neu- 
ronavigation. The previous skin incision was used, and a repeat craniotomy was performed, centered on the region of persistent connectivity. The dura was opened, the microscope was brought in, and the neuronavigation probe was used to identify the region of interest and the residual points of connectivity on DTI. A targeted disconnection was then performed using microneurosurgical technique. A postoperative external ventricular drain was then placed at the neurosurgeon's discretion.

\section{Seizure Outcome}

Seizure outcome was graded using the Engel outcome scale for epilepsy surgery. ${ }^{8}$

\section{Illustrative Cases \\ Case 1}

The patient presented with catastrophic epilepsy at age 3 months and was diagnosed with left hemimegalencephaly on MRI (Table 1). A left HD procedure was performed at 8 months of age. Seizures were not improved, and the child required 2 additional procedures resecting residual cortex, leaving the basal ganglia behind (Fig. 1). The child also required insertion of a ventriculoperitoneal (VP) shunt. Following these procedures, seizures were still not improved, the child's seizure outcome remained Engel Class IV, and he was receiving 4 antiseizure medications. His primary seizure type comprised complex partial seizures with secondary generalization. At age 15 years, 3-T MRI and DTI were performed and demonstrated persistent connectivity between a small remnant of basal frontal cortex and the corpus callosum (Fig. 1). A small frontal craniotomy was performed, and the area of suspected basal frontal connectivity was sectioned at the level of the corpus callosum using DTI-guided neuronavigation. The postoperative recovery was uneventful. Following this, the patient attained Engel Class II seizure outcome and was receiving 2 anticonvulsant medications at the 2-year follow-up.

\section{Case 4}

At the age of 6 years, the patient presented with sudden-onset intractable epilepsy. Workup and investigation, including MRI, suggested right Rasmussen's encephalitis. A temporal lobe biopsy was performed, the results of which supported the diagnosis. A right PIH was then performed, and the patient became seizure free for 7 years and was successfully weaned from his medications. At the age of 13 years, his seizures recurred and comprised complex partial seizures with suspected right frontal lobe onset. DTI sequences suggested a small region of persistent connectivity between the basal frontal lobe and the corpus callosum (Fig. 2). A second surgery was performed via a small frontal craniotomy, followed by resection of this band of connectivity, for which neuronavigation and microneurosurgical technique were used. No postoperative complications were noted. Following surgery, the child once again became seizure free, was weaned from his medications, and was Engel Class I at 2 years postoperatively.
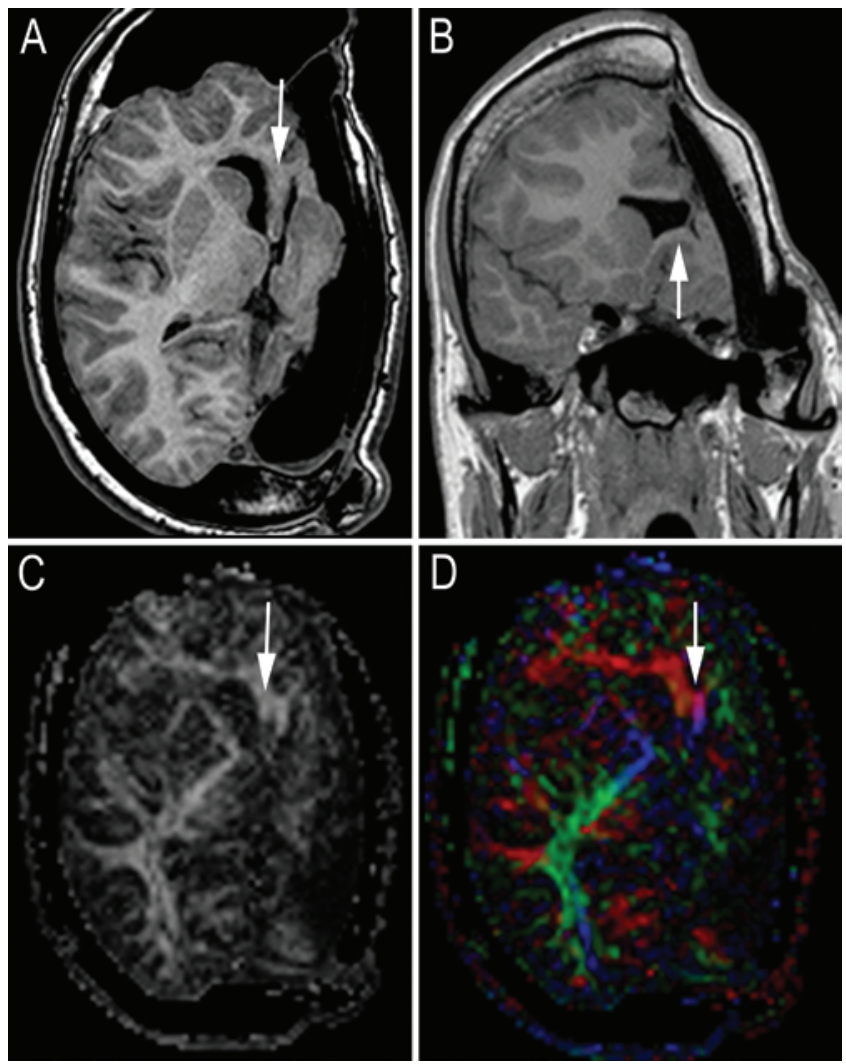

FIG. 1. Case 1. A 15-year-old boy with hemimegalencephaly and intractable epilepsy. Following 3 prior failed HD procedures to control his epilepsy, he underwent high-resolution volumetric 3-T MRI and DTI. A: Axial T1-weighted MRI demonstrating extent of prior HD procedures with preservation of the basal ganglia on the left side. Left VP shunt reservoir is seen. Arrow denotes area of suspected connectivity at the rostrum of the corpus callosum. B: Coronal T1-weighted MRI scan showing extent of left cranial vault deformity following HD procedures. Arrow indicates area of persistent connectivity between residual left posteroinferior mesial frontal lobe and the right normal hemisphere across the rostrum of the corpus callosum. C: Fractional anisotropy map from DTI shown on axial MRI at the same level of the rostrum of the corpus callosum as depicted in panel A. D: Color vector map of DTI at the same level as in panels $\mathrm{A}$ and $\mathrm{C}$, with red showing area of persistent crossing fibers at the level of the rostrum of the corpus callosum (arrows in C and D).

\section{Results}

Patient Demographic Data, Neuropathological Findings, and Initial Seizure Outcome

Between 1995 and 2012, 88 children underwent hemispherectomy for catastrophic epilepsy at the Hospital for Sick Children. Between 1995 and 1997, 8 patients underwent HD; whereas 80 patients underwent PIH between 1997 and 2012. In the time period of this study, 8 of 88 patients $(9.1 \%)$ were identified who experienced recurrent seizures after hemispherectomy, with suspected fiber tract connectivities between the 2 hemispheres. Two patients had prior HD procedures, whereas 6 had prior PIH. There were 3 males and 5 females. Initial seizure onset occurred at a median of 2.6 months (range newborn to 6 years). Initial PIH or HD was performed at a median age of 9.7 months (range 2-72 months). The neuropathological diagnoses included hemimegalencephaly $(n=4)$, hemispheric corti- 


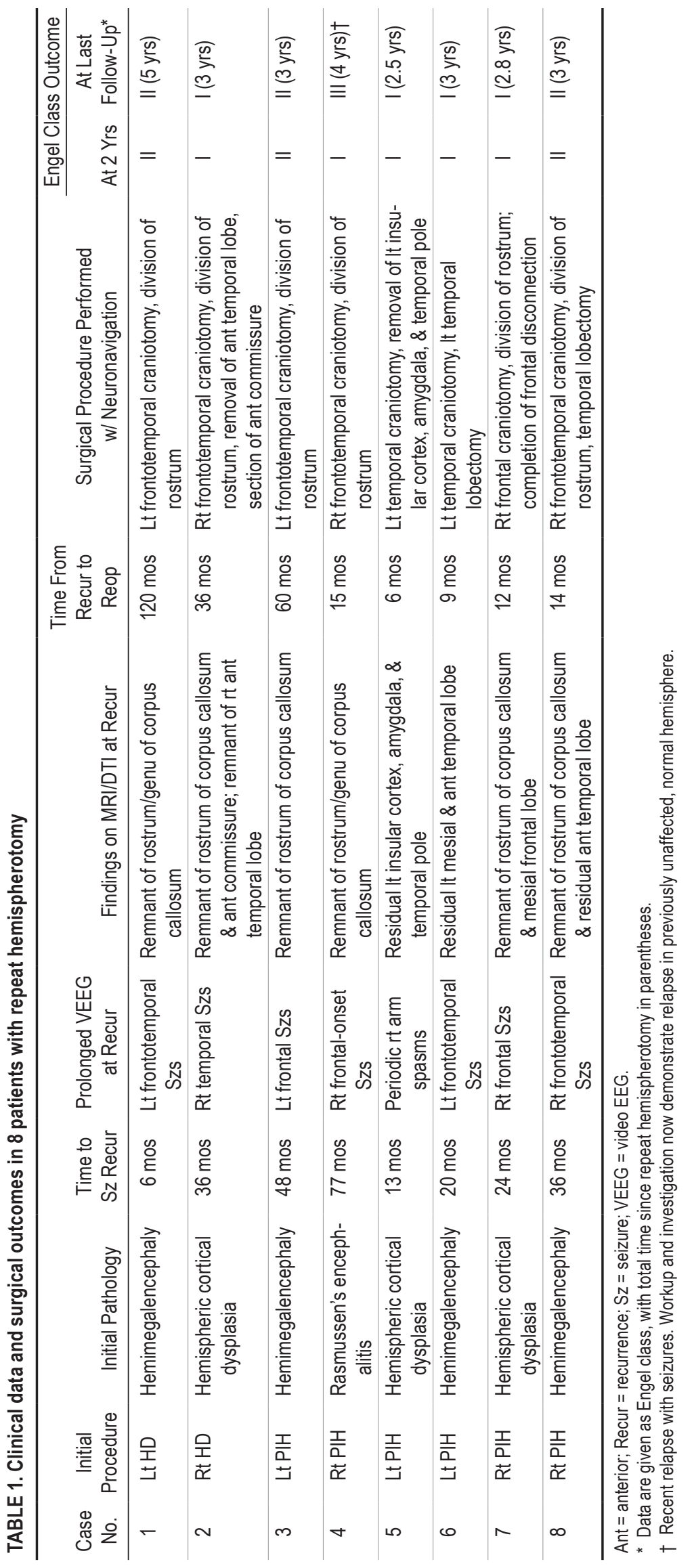




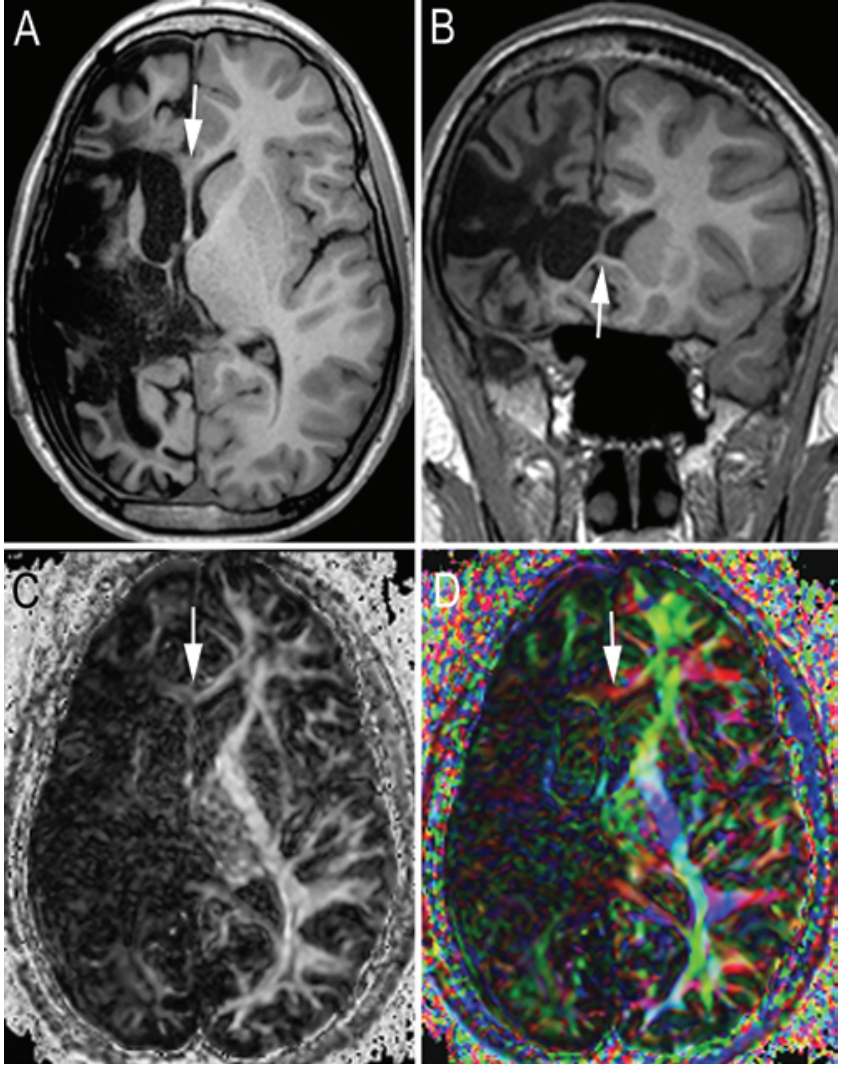

FIG. 2. Case 4. This 13-year-old boy was diagnosed with right hemispheric Rasmussen's encephalitis at age 6 years. At that time, he underwent a right $\mathrm{PIH}$. He became seizure free, and was weaned from all medications. Seizures recurred 7 years postoperatively, and high-resolution volumetric 3-T MRI and DTI were performed. A: Axial T1-weighted MRI showing characteristic atrophy of the affected hemisphere in Rasmussen's after PIH. Arrow indicates an area of suspected connectivity between the affected right and normal left hemispheres across the rostrum of the corpus callosum. B: Coronal T1-weighted MRI showing region of suspected connectivity between the 2 hemispheres through the rostrum of the corpus callosum (arrow). From this image, the right posteroinferior portion of the mesial frontal lobe is suspected to be connected to the left hemisphere. C: Fractional anisotropy map from DTI shown on axial MRI at the same level of the rostrum of the corpus callosum as depicted in panel A. D: Color vector map of DTI at the same level as in panels A and $\mathrm{C}$, with red showing area of persistent crossing fibers at the level of the rostrum of the corpus callosum (arrows in C and D).

cal dysplasia $(n=3)$, and Rasmussen's encephalitis $(n=1)$. There were 4 left- and 4 right-sided procedures. Seven patients were seizure free following their initial hemispheric procedure. The mean duration of seizure freedom after initial surgery was 32.5 months (range 6-77 months) for these 7 patients. Two of the 8 patients were treated with VP shunts following initial surgery, albeit in a delayed manner. Each patient had an expected hemiparesis after the first surgery that was worse than the preoperative status.

\section{Neurological, EEG, MEG, MRI, and DTI Investigations}

All 8 patients with recurrent seizures posthemispherectomy underwent detailed neurological examination, prolonged video EEG monitoring, and repeat neuroimaging with 1.5- to 3-T MRI and, in 4 cases, MEG. In all 8 patients, prolonged video EEG monitoring demonstrated persistent spike-wave disturbances from the surgically treated hemisphere coincident with clinical seizure onset. Careful attention was paid to the semiology of the seizure events to ensure that those captured were stereotypical and concordant with the other data from the workup. In all 8 patients, DTI demonstrated residual connectivity from the surgically treated hemisphere to the contralateral side across the corpus callosum from residual basal frontal lobe; across the anterior commissure from retained temporal lobe elements; or from demonstrated residual insular cortex (Table 1). An additional demonstration of the potential value of DTI in our case series is shown in Fig. 3 , which highlights areas of persistent connectivity not so clearly seen on T1-weighted MRI in another patient. MEG was performed in 4 patients; in 3 of them, spike clusters were found over the affected hemisphere in a distribution consistent with the suspected seizure onset zone.

\section{Operative Findings, Postoperative Course, and Seizure Outcome}

The median time from seizure recurrence to reoperation for the group was 14.5 months (range 6-120 months; mean 34 months). The median age at second surgery was 6.8 years (range 1.3-14 years). Average operative duration was 3 hours (range 1.8-4.3 hours) with a mean blood loss of $150 \mathrm{ml}$ (range 50-250 ml). One patient required a blood transfusion. The average hospital stay was 7 days (range 6-11 days). All patients were discharged home without further physiotherapy or rehabilitation requirements. There were no cases of infection or aseptic meningitis. There were no new cases of hydrocephalus or shunt failure. There were no new neurological deficits in any of the cases. One patient experienced an asymptomatic hemorrhagic conversion of a prior occipital lobe infarct. Five children were seizure free, and 3 had an Engel Class II outcome with a minimum follow-up of 24 months.

\section{Discussion}

In this series, we have shown that children with recurrent epilepsy after hemispheric disconnection procedures may benefit from reoperation in which surgery is targeted at demonstrated areas of persistent connectivities between the 2 hemispheres. Using a combination of repeat video EEG monitoring, MRI, MEG, DTI, and intraoperative neuronavigation, we have been successful at identifying residual white matter tract connectivities causing recurrent epilepsy. Transection of these residual white matter tracts has been a successful means by which these children can be rendered either seizure free or in a better seizure outcome state.

Children with catastrophic epilepsy typically fit into one of the main categories of disease processes that affect the brain and for which hemispherectomy is recommended. In this group of patients, one can expect a 70\%-85\% chance of seizure freedom following hemispherectomy. $3,5,9,12,14,15,18,19,27,30,31$ The technique of hemispherectomy has evolved over the past 30 years to include approaches that primarily disconnect the affected hemisphere from the contralateral side, and that have become more minimally invasive, requiring less cerebral tissue to be removed. Al- 

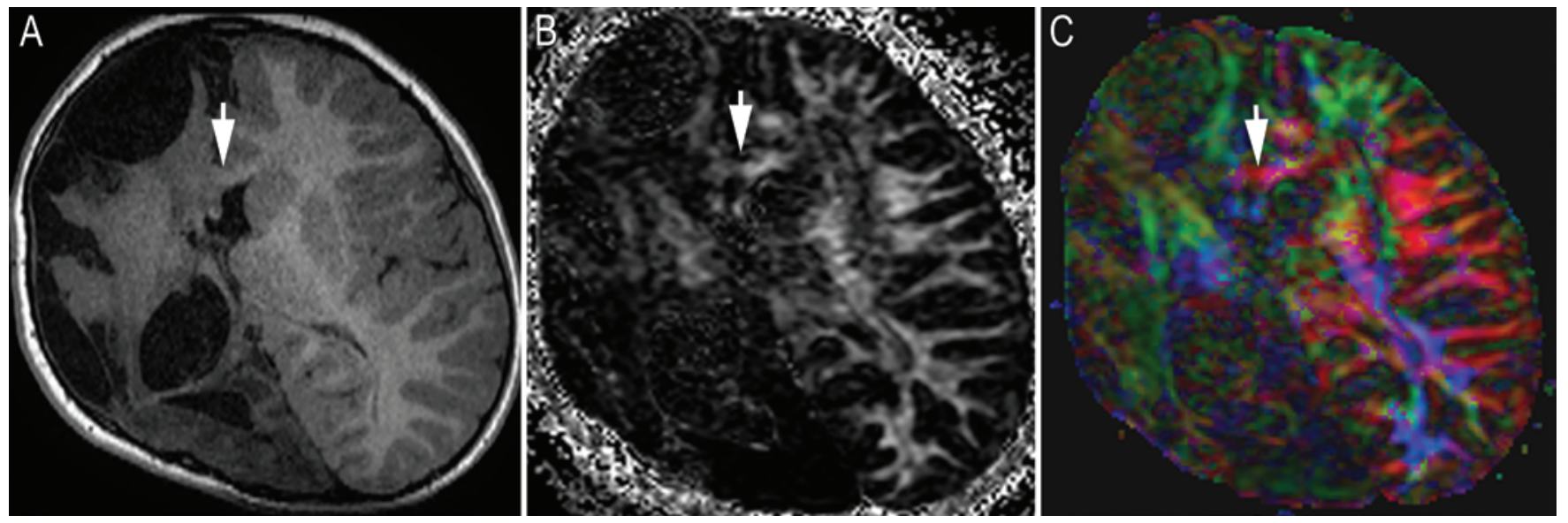

FIG. 3. Case 8. A 5-year-old girl with underlying right hemimegalencephaly at the time of repeat hemispherotomy for recurrent seizures. Following initial right PIH at age 3 years, the patient was free of seizures for 14 months. With the return of seizures, repeat imaging with MRI/DTI was performed. A: Axial T1-weighted MRI showing cranial vault asymmetry and distortion of the midline. It is difficult to ascertain the true location of the septum pellucidum. Arrow points to corpus callosum to the left of midline. B: Fractional anisotropy map from DTI at the same level as in panel A. Arrow points to the corpus callosum. C: Color vector map of DTI at the same level as in panels $A$ and $B$, with red showing area of persistent crossing fibers (arrow) that helps to confirm suspicions of persistent connectivity between the 2 hemispheres that were raised based on T1-weighted MR images. This region on DTI was targeted at redo craniotomy, using neuronavigation to sever the connections. It is now 3 years after repeat hemispherotomy and the patient has remained in Engel Class II in terms of seizure outcome.

though these advancements have led to expedited patient recovery and diminished length of stay in hospital, just as with anatomical hemispherectomy, there are instances of seizure recurrences or failures that arise after PIH or other functional disconnection procedures. Such patients with recurrent epilepsy after PIH can be problematic to investigate, and it can be difficult to make determinations regarding further treatments.

Recently, Vadera et al. described their experience with 36 patients who underwent reoperative hemispherotomy for diffuse unilateral hemispheric disease. ${ }^{30}$ These authors reported that prior to anatomical hemispherectomy, preoperative MRI studies showed that $44 \%$ of disconnections appeared to be complete, $44 \%$ appeared incomplete, and $11 \%$ were unknown. These investigators also showed that review of the MRI findings after PIH revealed the basal frontal and insular regions as the most likely sources of recurrent seizures, similar to the findings in our study. In their study, the majority of patients experienced improved seizure outcome on conversion of a PIH to an anatomical hemispherectomy. Finally, Vadera et al. determined that generalized ictal EEG activity before the repeat hemispherotomy and histopathological findings of cortical dysplasia correlated with a poorer prognosis in their patient population. ${ }^{30}$

Both HD and PIH allow for good to excellent seizure control while minimizing acute and long-term sequelae when compared with anatomical hemispherectomy. ${ }^{12}$ We have previously shown in our series of patients with hemispherectomy that PIH was superior to HD for control of catastrophic epilepsy. We have also characterized the relevance of spike clusters in children undergoing hemispherectomy. ${ }^{29}$ In this study, we have shown that MEG performed at recurrence of seizures posthemispherectomy can be used as additive information together with prolonged video EEG recordings and MRI/DTI. The po- tential true value of EEG and MEG is in determining the unilateral nature of the epileptic disturbances to the affected hemisphere and excluding patients who may possibly exhibit bihemispheric disturbances.

Use of DTI can supplement detailed anatomical imaging to identify residual white matter connections that could be responsible for seizure propagation. That being stated, tractography has been of considerable value for neurosurgeons embarking on intraaxial brain tumor surgeries. In this situation, DTI allows for the visualization of the relationship between a neoplasm and the surrounding white matter tracts for both preoperative planning and intraoperative resection when combined with neuronavigation. However, the use of DTI in epilepsy surgery has been limited to a few descriptive studies.,13,22,28 Toda et al. described a case of a 5-year-old boy who underwent PIH for hemispheric epilepsy resulting from diffuse hemispheric cortical dysplasia..$^{28}$ Three months after hemispherotomy, the child developed recurrent seizures. MRI and DTI were performed during follow-up, and these studies demonstrated residual crossing fibers at the genu of the corpus callosum. On further resection of the genu in this case, the patient became seizure free once again, although the length of follow-up was not provided. In the current study, DTI was of value in identifying persistent white matter tracts, including the rostrum/genu of the corpus callosum and the anterior commissure, which connects bilateral temporal lobes. Resection of the remnant temporal lobe would effectively remove the connection with the normal temporal lobe. It should be stated here that thin-slice T1-weighted images, particularly in the coronal plane, are also of considerable value in evaluating the persistence of connectivities between the 2 hemispheres.

Neuronavigation is used routinely in neurosurgery in the developed world. We have been using neuronavigation for complex pediatric neurosurgical procedures since 
$1992.1,6,7,11,23,25$ In the present series, neuronavigation was extremely helpful and reliable in delineating the residual white matter tracts of connectivity across the corpus callosum and anterior commissure. The neuroanatomy of the lateral ventricle, the anterior cerebral and pericallosal arteries, and the interhemispheric fissure may be somewhat distorted at the time of repeat hemispherotomy-hence the value of a tracking system such as neuronavigation.

Having studied our failed cases in detail, we conclude that the main reason for failure was incomplete resection of the rostrum/genu of the corpus callosum, especially at its most inferior and anterior segment. Although the pericallosal arteries serve as valuable and important landmarks for marking the midline approach to sectioning the corpus callosum during $\mathrm{PIH}$, these arteries deviate from the corpus callosum the more proximally the neurosurgeon follows them toward the $\mathrm{A}_{2}$ and $\mathrm{A}_{1}$ segments of the anterior cerebral artery. The second most common reason for failure was the presence of residual temporal lobe with a persistent anterior commissure. Based on our experience, it is therefore critical during initial PIH surgery that the corpus callosum at its rostrum be sectioned carefully and completely to ensure that disconnection has been achieved.

Interestingly, many of our patients were seizure free for several years prior to the recurrence of their seizures. This may reflect the fact that disrupting the vast majority of connections between the 2 hemispheres renders a patient in a seizure-free state after incomplete hemispherotomy. Over time, and with the continued presence of neuropathologically and electrically abnormal cerebral tissue and networks in the retained hemisphere, alternate epileptogenic pathways may develop, leading to the onset of recurrent epilepsy. The median time from seizure recurrence to reoperation for the group was 14.5 months. This relatively long time to repeat disconnection reflects the time taken to adjust anticonvulsant medications in some cases, and the time to repeat the epilepsy surgery workup, including video EEG and MEG, among other studies.

Despite the repeat hemispherotomies we performed in this series, there were 3 patients $(37.5 \%)$ who continued to have seizures even though we were satisfied with our determination that the hemispheric disconnection was now complete. As in the case series by Vadera et al. in which there was a $36 \%$ failure rate of anatomical hemispherectomy after PIH, we have concluded that these cases may well represent true bihemispheric epilepsy. ${ }^{30}$

In the future, it would be interesting and important to conduct a prospective study in which DTI is used postoperatively in all patients following PIH to assess the extent of disconnection and to determine how many patients will demonstrate seizure recurrence with or without identifiable tracts of residual connectivity between the hemispheres.

\section{Conclusions}

The options available to children who have undergone $\mathrm{PIH}$ or other functional hemispheric procedures for catastrophic epilepsy are somewhat limited. Here, we provide our experience with a series of patients in whom recurrent epilepsy was traced back to persistent white matter tracts that were not identified or appreciated at the time of original surgery. We suggest that it is an option to consider repeat hemispherotomy in these patients, provided that the seizure semiology and the electrophysiological and neuroimaging studies are concordant. Conversion to an anatomical hemispherectomy may not always be necessary in this population of patients. We have shown that repeat surgery to ensure that a complete disconnection has been accomplished carries an acceptable risk and is straightforward and beneficial in the majority of our patients. In these children, our efforts provided them with a second opportunity to achieve long-term good seizure control.

\section{Acknowledgments}

This study was made possible through support from the Jack Beqaj fund and from the Wiley Family fund within the Sick Kids Hospital Foundation.

\section{References}

1. Alshail E, Rutka JT, Drake JM, Hoffman HJ, Humphreys $\mathrm{R}$, Phillips J, et al: Utility of frameless stereotaxy in the resection of skull base and Basal cerebral lesions in children. Skull Base Surg 8:29-38, 1998

2. Bahuleyan B, Robinson S, Nair AR, Sivanandapanicker JL, Cohen AR: Anatomic hemispherectomy: historical perspective. World Neurosurg 80:396-398, 2013

3. Beier AD, Rutka JT: Hemispherectomy: historical review and recent technical advances. Neurosurg Focus 34(6):E11, 2013

4. Choudhri AF, Whitehead MT, McGregor AL, Einhaus SL, Boop FA, Wheless JW: Diffusion tensor imaging to evaluate commissural disconnection after corpus callosotomy. Neuroradiology 55:1397-1403, 2013

5. Delalande O, Bulteau C, Dellatolas G, Fohlen M, Jalin C, Buret V, et al: Vertical parasagittal hemispherotomy: surgical procedures and clinical long-term outcomes in a population of 83 children. Neurosurgery 60 (2 Suppl 1):ONS19ONS32, 2007

6. Drake JM, Prudencio J, Holowaka S, Rutka JT, Hoffman HJ, Humphreys RP: Frameless stereotaxy in children. Pediatr Neurosurg 20:152-159, 1994

7. Drake JM, Rutka JT, Hoffman HJ: ISG viewing wand system. Neurosurgery 34:1094-1097, 1994

8. Engel J Jr, Van Ness PC, Rasmussen T, Ojemann LM: Outcome with respect to epileptic seizures, in Engel Jr J (ed): Surgical Treatment of the Epilepsies. New York: Raven Press, 1993, pp 609-621

9. Hoffman HJ, Hendrick EB, Dennis M, Armstrong D: Hemispherectomy for Sturge-Weber syndrome. Childs Brain 5:233-248, 1979

10. Ibrahim GM, Rutka JT: Recent advances in epilepsy surgery in children, in Basant M, Laws ER, Kaye AH (eds): Current Progress in Neurosurgery. Mumbai: Kothari Medical Subscription Services Pvt Ltd, 2014, Vol 1, pp 1-16

11. Jea A, Vachhrajani S, Johnson KK, Rutka JT: Corpus callosotomy in children with intractable epilepsy using frameless stereotactic neuronavigation: 12-year experience at the Hospital for Sick Children in Toronto. Neurosurg Focus 25(3):E7, 2008

12. Kwan A, Ng WH, Otsubo H, Ochi A, Snead OC III, Tamber MS, et al: Hemispherectomy for the control of intractable epilepsy in childhood: comparison of 2 surgical techniques in a single institution. Neurosurgery 67 (2 Suppl Operative):429-436, 2010

13. Lee MJ, Kim HD, Lee JS, Kim DS, Lee SK: Usefulness of diffusion tensor tractography in pediatric epilepsy surgery. Yonsei Med J 54:21-27, 2013 
14. Lew SM, Matthews AE, Hartman AL, Haranhalli N: Posthemispherectomy hydrocephalus: results of a comprehensive, multiinstitutional review. Epilepsia 54:383-389, 2013

15. Moosa AN, Jehi L, Marashly A, Cosmo G, Lachhwani D, Wyllie E, et al: Long-term functional outcomes and their predictors after hemispherectomy in 115 children. Epilepsia 54:1771-1779, 2013

16. Moseley ME, Cohen Y, Kucharczyk J, Mintorovitch J, Asgari HS, Wendland MF, et al: Diffusion-weighted MR imaging of anisotropic water diffusion in cat central nervous system. Radiology 176:439-445, 1990

17. Ogunmekan AO, Hwang PA, Hoffman HJ: Sturge-WeberDimitri disease: role of hemispherectomy in prognosis. Can J Neurol Sci 16:78-80, 1989

18. Peacock WJ: Hemispherectomy for the treatment of intractable seizures in childhood. Neurosurg Clin N Am 6:549-563, 1995

19. Peacock WJ, Wehby-Grant MC, Shields WD, Shewmon DA, Chugani HT, Sankar R, et al: Hemispherectomy for intractable seizures in children: a report of 58 cases. Childs Nerv Syst 12:376-384, 1996

20. Phung J, Krogstad P, Mathern GW: Etiology associated with developing posthemispherectomy hydrocephalus after resection-disconnection procedures. J Neurosurg Pediatr 12:469-475, 2013

21. Pierpaoli C, Jezzard P, Basser PJ, Barnett A, Di Chiro G: Diffusion tensor MR imaging of the human brain. Radiology 201:637-648, 1996

22. Radhakrishnan A, James JS, Kesavadas C, Thomas B, Bahuleyan B, Abraham M, et al: Utility of diffusion tensor imaging tractography in decision making for extratemporal resective epilepsy surgery. Epilepsy Res 97:52-63, 2011

23. Rutka JT, Souweidane M, terBrugge K, Armstrong D, Zuker $\mathrm{R}$, Clarke H, et al: Separation of craniopagus twins in the era of modern neuroimaging, interventional neuroradiology, and frameless stereotaxy. Childs Nerv Syst 20:587-592, 2004

24. Sorensen AG, Wu O, Copen WA, Davis TL, Gonzalez RG, Koroshetz WJ, et al: Human acute cerebral ischemia: detection of changes in water diffusion anisotropy by using MR imaging. Radiology 212:785-792, 1999

25. Stapleton SR, Kiriakopoulos E, Mikulis D, Drake JM, Hoffman HJ, Humphreys R, et al: Combined utility of functional MRI, cortical mapping, and frameless stereotaxy in the resection of lesions in eloquent areas of brain in children. Pediatr Neurosurg 26:68-82, 1997

26. Sugimoto T, Otsubo H, Hwang PA, Hoffman HJ, Jay V,
Snead OC III: Outcome of epilepsy surgery in the first three years of life. Epilepsia 40:560-565, 1999

27. Terra-Bustamante VC, Inuzuka LM, Fernandes RM, EscorsiRosset S, Wichert-Ana L, Alexandre V Jr, et al: Outcome of hemispheric surgeries for refractory epilepsy in pediatric patients. Childs Nerv Syst 23:321-326, 2007

28. Toda K, Baba H, Ono T, Ono K: The utility of diffusion tensor imaging tractography for post-operative evaluation of a patient with hemispherotomy performed for intractable epilepsy. Brain Dev 36:641-644, 2014

29. Torres CV, Fallah A, Ibrahim GM, Cheshier S, Otsubo H, Ochi A, et al: The role of magnetoencephalography in children undergoing hemispherectomy. J Neurosurg Pediatr 8:575-583, 2011

30. Vadera S, Moosa AN, Jehi L, Gupta A, Kotagal P, Lachhwani $\mathrm{D}$, et al: Reoperative hemispherectomy for intractable epilepsy: a report of 36 patients. Neurosurgery 71:388-393, 2012

31. Villemure JG, Daniel RT: Peri-insular hemispherotomy in paediatric epilepsy. Childs Nerv Syst 22:967-981, 2006

32. Villemure JG, Mascott CR: Peri-insular hemispherotomy: surgical principles and anatomy. Neurosurgery 37:975-981, 1995

\section{Disclosures}

The authors report no conflict of interest concerning the materials or methods used in this study or the findings specified in this paper.

\section{Author Contributions}

Conception and design: Rutka, Kiehna. Acquisition of data: Rutka, Kiehna, Widjaja, Holowka, Ochi, Thompson. Analysis and interpretation of data: Rutka, Kiehna, Widjaja, Holowka, Snead, Ochi, Thompson. Drafting the article: Rutka, Kiehna. Critically revising the article: all authors. Reviewed submitted version of manuscript: all authors. Approved the final version of the manuscript on behalf of all authors: Rutka. Study supervision: Rutka, Widjaja.

\section{Correspondence}

James T. Rutka, Division of Neurosurgery, Ste. 1503, Hospital for Sick Children, 555 University Ave., Toronto, ON M5G 1X8, Canada. email: james.rutka@sickkids.ca. 